Pacific

Journal of

Mathematics

ASSOCIATED PRIMES OF LOCAL COHOMOLOGY MODULES OVER REGULAR RINGS

TONY J. PUTHENPURAKAL

Volume $282 \quad$ No. 1

May 2016 


\title{
ASSOCIATED PRIMES OF LOCAL COHOMOLOGY MODULES OVER REGULAR RINGS
}

\author{
TONY J. PUTHENPURAKAL
}

\begin{abstract}
Let $R$ be an excellent regular ring of dimension $d$ containing a field $K$ of characteristic zero. Let $I$ be an ideal in $R$. We show that Ass $H_{I}^{d-1}(R)$ is a finite set. As an application, we show that if $I$ is an ideal of height $g$ with height $Q=g$ for all minimal primes of $I$ then for all but finitely many primes $P \supseteq I$ with height $P \geq g+2$, the topological space $\operatorname{Spec}^{\circ}\left(R_{P} / I R_{P}\right)$ is connected. We also show that to prove a conjecture of Lyubeznik (regarding finiteness of associate primes for local cohomology modules) for all excellent regular rings of dimension $\leq \boldsymbol{d}$ containing a field of characteristic zero, it suffices to prove $\operatorname{Ass}_{S} H_{J}^{g+1}(S)$ is finite for all ideals $J$ in $S$ of height $g$ (here $0 \leq g \leq d$ ), where $S$ is an excellent regular domain of dimension $\leq d$ containing an uncountable field of characteristic zero.
\end{abstract}

\section{Introduction}

Throughout this paper $R$ is a commutative Noetherian ring. If $M$ is an $R$-module and if $I$ is an ideal in $R$, we denote by $H_{I}^{i}(M)$ the $i$-th local cohomology module of $M$ with respect to $I$.

The following conjecture is due to Lyubeznik [2002]:

Conjecture 1.1. If $R$ is a regular ring, then each local cohomology module $H_{I}^{i}(R)$ has finitely many associated prime ideals.

There are many cases where this conjecture is true: for regular rings $R$ of prime characteristic [Huneke and Sharp 1993], for regular local and affine rings of characteristic zero [Lyubeznik 1993], and for unramified regular local rings of mixed characteristic [Lyubeznik 2000]. It is also true for smooth $\mathbb{Z}$-algebras [Bhatt et al. 2014].

Lyubeznik [2002] especially asked whether Conjecture 1.1 is valid for a regular ring $R$ containing a field of characteristic zero. It is easy to give examples where existing techniques, to show finiteness of associate primes of local cohomology modules, fail: 
Example 1.2. (a) Let $(S, \mathfrak{m})$ be a complete local domain of dimension $d \geq 2$ containing a field of characteristic zero. Assume $S$ is not regular. Let the singular locus be defined by the ideal $J$. Notice $J \neq 0$. Let $x \in J$ be nonzero. Set $R=S_{x}$. Then $R$ is a domain of dimension $d-1$; see [Matsumura 1989, Lemma 1, p. 247]. Also clearly $R$ is regular.

(b) Let $T$ be a regular domain as above containing a field $K$ of characteristic zero. Let $f \in K\left[X_{1}, \ldots, X_{n}\right]$ be a smooth polynomial. Then $R=T\left[X_{1}, \ldots, X_{n}\right] /(f)$ is a regular ring.

In both the examples above, we do not know whether $\operatorname{Ass}_{R}\left(H_{I}^{i}(R)\right)$ is a finite set for all ideals $I$ of $R$.

In all the essential cases where finiteness of associated primes is known, the local cohomology modules of $R$ have some additional global structure. If $R$ is of characteristic $p$ then local cohomology modules have the structure of $F$-modules [Lyubeznik 1997]. In characteristic zero, for complete local rings and smooth affine algebras over algebraically closed fields, local cohomology modules have an appropriate $D$-module structure. For smooth $\mathbb{Z}$-algebras, Bhatt et al. [2014] use a rather clever mixture of $D$-module and $F$-module theory. For a general regular ring containing a field of characteristic zero, there is no obvious structure that local cohomology modules satisfy that we can exploit to prove finiteness of associate primes.

For the rest of the paper, assume that $R$ contains a field of characteristic zero. For simplicity, we assume that $\operatorname{dim} R=d$ is finite. By Grothendieck's vanishing theorem, $H_{I}^{i}(R)=0$ for all $i>d$; see [Brodmann and Sharp 1998, Theorem 6.1.2]. In general, for a Noetherian ring $R$ of dimension $d$, the set $\operatorname{Ass}_{R}\left(H_{I}^{d}(R)\right)$ is finite; see [Brodmann et al. 2000, Remark 3.11] (also see [Marley 2001, Proposition 2.3]). If $R$ is a regular ring of dimension $d$ and $I$ is an ideal in $R$ then using the HartshorneLichtenbaum theorem (cf. [Iyengar et al. 2007, Theorem 14.1]), it is easy to prove that

$$
\operatorname{Ass}_{R}\left(H_{I}^{d}(R)\right)=\{P \mid P \in \operatorname{Min} R / I \text { and height } P=d\} .
$$

The following is the main result of this paper:

Theorem 1.3. Let $R$ be an excellent regular ring of dimension d containing a field of characteristic zero. Let I be an ideal in $R$. Then $\operatorname{Ass}_{R}\left(H_{I}^{d-1}(R)\right)$ is a finite set.

The main idea of this paper is that it is fruitful to look at the following relative situation: Let $I \supseteq J$ be ideals in a Noetherian ring $R$. We have a natural map $\theta_{I, J}^{i}: H_{I}^{i}(R) \rightarrow H_{J}^{i}(R)$ for each $i \geq 0$. Fix $i_{0} \geq 0$. Set

$$
\mathcal{C}_{R}^{i_{0}}=\left\{(I, J) \mid I \supset J \text { and } \sharp \operatorname{Ass}_{R}\left(\text { image } \theta_{I, J}^{i_{0}}\right)=\infty\right\} .
$$

Here $\sharp S$ denotes the number of elements in a set $S$. If $\operatorname{Ass}_{R} H_{I}^{i_{0}}(R)$ is infinite then $(I, I) \in \mathcal{C}_{R}^{i_{0}}$. Conversely, if $(I, J) \in \mathcal{C}_{R}^{i_{0}}$ then $\operatorname{Ass}_{R} H_{J}^{i_{0}}(R)$ is infinite. We partially $\operatorname{order} \mathcal{C}_{R}^{i_{0}}$ as follows: set $(I, J) \preceq\left(I^{\prime}, J^{\prime}\right)$ if $I^{\prime} \supseteq I$ and $J^{\prime} \supseteq J$. It is easy to see 
that every ascending chain in $\mathcal{C}_{R}^{i_{0}}$ stabilizes. If $\mathcal{C}_{R}^{i_{0}}$ is nonempty then its maximal elements have some peculiar properties; see Lemma 2.10.

The following result is a crucial ingredient in the proof of Theorem 1.3.

Theorem 1.4. Let $R$ be an excellent regular domain of dimension d containing an uncountable field of characteristic zero. Assume for some $i_{0}$ that the set $\mathcal{C}_{R}^{i_{0}}$ is nonempty. Let $(I, J)$ be a maximal element in $\mathcal{C}_{R}^{i_{0}}$. Then there exists a multiplicatively closed set $S$ of $R$ such that in the ring $A=S^{-1} R$ we have

(a) $\left(S^{-1} I, S^{-1} J\right)$ is a maximal element in $\mathcal{C}_{A}^{i_{0}}$,

(b) height $S^{-1} I=i_{0}$,

(c) $S^{-1} I=P_{1} \cap P_{2} \cap \cdots \cap P_{r}$, where $P_{i}$ is a prime in $A$ of height $i_{0}$,

(d) Ass image $\theta_{S^{-1} I, S^{-1} J}^{i_{0}} \supseteq \mathrm{m}-\operatorname{Spec}(A)$,

(e) height $\mathfrak{m}=$ height $\mathfrak{m}^{\prime}$ for $\mathfrak{m}, \mathfrak{m}^{\prime} \in \mathfrak{m}-\operatorname{Spec}(A)$,

(f) $\mathrm{m}-\mathrm{Spec}(A)$ is a countably infinite set.

Furthermore, if $\operatorname{Ass}_{R}\left(H_{L}^{r}(R)\right)$ is a finite set for all $r<i_{0}$ and for all ideals $L$ of $R$ then height $S^{-1} J=i_{0}-1$.

The assumptions that $R$ is a domain and contains an uncountable field are mild hypotheses; see Section 3. The assumption on the excellence of $R$ is satisfied by most examples. As an easy consequence of Theorem 1.4, we get the following significant simplification of Lyubeznik's conjecture.

Theorem 1.5. The following are equivalent:

(i) Lyubeznik's conjecture has a positive answer for all excellent regular rings of dimension $\leq d$ containing a field of characteristic zero.

(ii) For all excellent regular domains $R$ of dimension $\leq d$ containing an uncountable field of characteristic zero, $\operatorname{Ass}_{R}\left(H_{J}^{g+1}(R)\right)$ is a finite set for all ideals $J$ of height $g$, with $1 \leq g \leq d$.

The following are applications of Theorem 1.3:

Corollary 1.6. Let $R$ be an excellent regular ring of dimension $d \leq 4$ containing a field $K$. Then for any ideal $I$, we have Ass $H_{I}^{i}(R)$ is a finite set for all $i \geq 0$.

If $M$ is an $R$-module then set

$$
\operatorname{Ass}_{R}^{i}(M)=\{P \mid P \in \text { Ass } M \text { and height } P=i\} .
$$

Using the Hartshorne-Lichtenbaum theorem, we get that if $R$ is regular and $I$ is an ideal in $R$ then

$$
\bigcup_{i \geq 0} \operatorname{Ass}_{R}^{i}\left(H_{I}^{i}(R)\right)=\operatorname{Min} R / I
$$

see Corollary 8.2. 
Corollary 1.7. Let $R$ be an excellent regular ring of dimension d containing a field of characteristic zero. Let I be an ideal in $R$. Then

$$
\bigcup_{i \geq 0} \operatorname{Ass}_{R}^{i+1}\left(H_{I}^{i}(R)\right) \text { is a finite set. }
$$

We need a description of primes which appear in Corollary 1.7. To do so, we first make the following definition: recall if $(A, \mathfrak{m})$ is a local ring then $\operatorname{Spec}^{\circ}(A)=$ $\operatorname{Spec}(A) \backslash\{\mathfrak{m}\}$ considered as a subspace of $\operatorname{Spec}(A)$.

Definition 1.8. Let $(A, \mathfrak{m})$ be a local ring and let $I$ be an ideal in $A$. We say $\operatorname{Spec}^{\circ}(A / I)$ is absolutely connected if for every flat local map $(A, \mathfrak{m}) \rightarrow(B, \mathfrak{n})$ with $\mathfrak{m} B=\mathfrak{n}, B$ complete and $B / \mathfrak{n}$ algebraically closed, $\operatorname{Spec}^{\circ}(B / I B)$ is connected.

Remark 1.9. It is easy to see that if $\operatorname{Spec}^{\circ}(A / I)$ is absolutely connected then it is connected.

As a consequence of a result of Ogus [1973, Corollary 2.11] (also see [Huneke and Lyubeznik 1990, Theorem 1.1]), we get this:

Proposition 1.10. Let $R$ be an excellent regular ring of dimension d containing a field of characteristic zero and let $I$ be an ideal in $R$ of height $g$. Assume height $Q=g$ for all minimal primes $Q$ of $I$. Then

$$
\begin{aligned}
& \bigcup_{i \geq 0} \operatorname{Ass}_{R}^{i+1}\left(H_{I}^{i}(R)\right)=\{P \mid P \supseteq I, \text { height } P \geq g+2, \text { and } \\
&\left.\operatorname{Spec}^{\circ}\left(R_{P} / I_{P}\right) \text { is NOT absolutely connected }\right\} .
\end{aligned}
$$

As an immediate application we get this:

Corollary 1.11. Assume the hypotheses of Proposition 1.10 hold. For all but finitely many primes $P \supseteq I$ with height $P \geq g+2$, we get that $\operatorname{Spec}^{\circ}\left(R_{P} / I_{P}\right)$ is absolutely connected. In particular, $\operatorname{Spec}^{\circ}\left(R_{P} / I_{P}\right)$ is connected.

Here is an overview of the contents of the paper. In Section 2 we discuss a few general results that we need. In the next section we discuss the flat extension $R \rightarrow R[[X]]_{X}$. We need it as an essential technique in our paper requires an uncountable field contained in $R$. In Section 4 we discuss countable prime avoidance. We also give a construction which is used several times in our paper. In Section 5 we prove Theorem 1.4. In the next section we prove our main result, Theorem 1.3. In Section 7 we prove the simplicity of a $D$-module. This is needed in the proof of Theorem 1.3. In Section 8 we prove Corollary 1.6. Finally in Section 9 we give proofs of Corollary 1.7 and Proposition 1.10.

\section{Generalities}

In this section we prove some general results. Some of them are perhaps already known to the experts. However, we prove them as we do not have a reference. 
We first prove the following general result:

Proposition 2.1. Let $R$ be a Noetherian ring and let $I$ be an ideal in $R$. Let $M$ be a finitely generated $R$-module. Then $\operatorname{Ass}_{R}\left(H_{I}^{i}(M)\right)$ is a countable set.

To prove the proposition, we need the following notion.

Definition 2.2. We say an $R$-module $E$ is countably generated if there exists a countable set of elements $\left\{e_{n}\right\}_{n \geq 1}$ which generate $E$ as an $R$-module.

The following lemma is useful:

Lemma 2.3. Let $R$ be a Noetherian ring and let $E$ be a countably generated $R$-module. Then $\operatorname{Ass}_{R}(E)$ is a countable set.

Proof. Let $\left\{e_{n}\right\}_{n \geq 1}$ generate $E$ as an $R$-module. For $m \geq 1$, let $D_{m}$ be the $R$-submodule of $E$ generated by $e_{1}, \ldots, e_{m}$. Clearly $D_{m} \subseteq D_{m+1}$ for all $m \geq 1$, $E=\bigcup_{m \geq 1} D_{m}$ and $\bigcup_{m \geq 1} \operatorname{Ass}_{R}\left(D_{m}\right) \subseteq \operatorname{Ass}_{R}(E)$. If $P \in \operatorname{Ass}_{R}(E)$ then $P=(0: u)$ for some $u \in E$. Say $u \in D_{r}$. Then $P \in \operatorname{Ass}_{R}\left(D_{r}\right)$. Thus

$$
\bigcup_{m \geq 1} \operatorname{Ass}_{R}\left(D_{m}\right)=\operatorname{Ass}_{R}(E) \text {. }
$$

Since $R$ is Noetherian and $D_{m}$ is a finitely generated $R$-module, $\operatorname{Ass}_{R}\left(D_{m}\right)$ is a finite set for all $m \geq 1$. It follows that $\operatorname{Ass}_{R}(E)$ is a countable set.

Proof of Proposition 2.1. Fix $i \geq 0$. Then

$$
H_{I}^{i}(M)=\underline{\lim _{\longrightarrow}} \operatorname{Ext}_{R}^{i}\left(R / I^{n}, M\right) .
$$

In particular $H_{I}^{i}(M)$ is a quotient of $\bigoplus_{n \geq 1} \operatorname{Ext}_{R}^{i}\left(R / I^{n}, M\right)$. It follows that $H_{I}^{i}(M)$ is countably generated. The result now follows from Lemma 2.3.

2.4. We need to compare $R$-linear maps $f, g: E \rightarrow F$, where $E, F$ are $R$-modules. Definition 2.5. Let $f, g: E \rightarrow F$ be $R$-linear maps. We say $f \cong g$ if there exist isomorphisms $\alpha: E \rightarrow E$ and $\beta: F \rightarrow F$ such that the following diagram commutes:

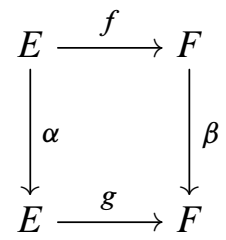

The following result is clear:

Proposition 2.6. Let $R$ be a ring and let $E, F$ be $R$-modules. Let $f, g: E \rightarrow F$ be $R$-linear. If $f \cong g$ then we have the following isomorphisms of $R$-modules:

ker $f \cong \operatorname{ker} g, \quad$ image $f \cong$ image $g$ and coker $f \cong$ coker $g$. 
2.7. Let $R$ be a Noetherian ring and let $I, J$ be ideals of $R$ with $I \supseteq J$. Let $\Gamma_{I}, \Gamma_{J}$ be the $I$-torsion and $J$-torsion functors respectively. Let $M$ be an $R$-module. Let us recall the construction of the natural maps

$$
\theta_{I, J}^{i}(M): H_{I}^{i}(M) \rightarrow H_{J}^{i}(M)
$$

for all $i \geq 0$ :

Let $\mathbb{E}$ be an injective resolution of $M$. Then note that we have a natural morphism of complexes $\theta: \Gamma_{I}(\mathbb{E}) \rightarrow \Gamma_{J}(\mathbb{E})$. Taking cohomology, we obtain our natural maps $\theta_{I, J}^{i}(M)$ for all $i \geq 0$.

The following result will be used several times.

Lemma 2.8. Let $R \rightarrow S$ be a flat map of Noetherian rings. Let $I, J$ be ideals of $R$ with $I \supseteq J$. Let $M$ be an $R$-module. Then for all $i \geq 0$, we have

$$
\theta_{I, J}^{i}(M) \otimes S \cong \theta_{I S, J S}^{i}(M \otimes S) .
$$

Proof. Let $\mathbb{E}$ be an injective resolution of $M$. Then $\mathbb{E} \otimes S$ is $\Gamma_{K S}$-acyclic for any ideal $K$ of $R$; see [Brodmann and Sharp 1998, Theorem 4.1.9]. Furthermore, we have a natural equivalence of functors $\Gamma_{K} \otimes S \cong \Gamma_{K S}$; see [loc. cit., Proposition 4.3.1]. Thus we have a commutative diagram of complexes

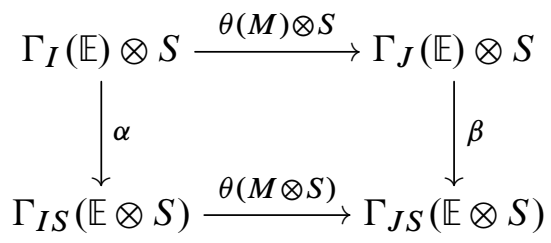

where $\alpha$ and $\beta$ are isomorphisms of complexes. The result follows.

2.9. Let $R$ be a Noetherian ring. Let $I \supseteq J$ be ideals in $R$. We have a natural map $\theta_{I, J}^{i}: H_{I}^{i}(R) \rightarrow H_{J}^{i}(R)$ for each $i \geq 0$. Fix $i_{0} \geq 0$. Set

$$
\mathcal{C}_{R}^{i_{0}}=\left\{(I, J) \mid I \supset J \text { and } \sharp \operatorname{Ass}_{R}\left(\text { image } \theta_{I, J}^{i_{0}}\right)=\infty\right\} .
$$

Here $\sharp S$ denotes the number of elements in a set $S$. We partially order $\mathcal{C}_{R}^{i_{0}}$ as follows: set $(I, J) \preceq\left(I^{\prime}, J^{\prime}\right)$ if $I^{\prime} \supseteq I$ and $J^{\prime} \supseteq J$. It is easy to see that every ascending chain in $\mathcal{C}_{R}^{i_{0}}$ stabilizes. If $\mathcal{C}_{R}^{i_{0}}$ is nonempty then its maximal element has a peculiar property which we now describe:

Lemma 2.10. Assume the hypotheses of 2.9 hold. Assume $(I, J) \in \mathcal{C}_{R}^{i_{0}}$ is a maximal element. Let $S$ be a multiplicatively closed subset of $R$. If $\left(S^{-1} I, S^{-1} J\right) \in \mathcal{C}_{S^{-1} R}^{i_{0}}$ then

(1) $\left(S^{-1} I, S^{-1} J\right)$ is a maximal element in $\mathcal{C}_{S^{-1} R}^{i_{0}}$,

(2) $S^{-1} I \cap R=I$ and $S^{-1} J \cap R=J$. 
To prove the lemma, we need the following easy result.

Proposition 2.11. Assume the hypotheses of Lemma 2.10 hold. If $(K, L) \in \mathcal{C}_{S^{-1} R}^{i_{0}}$ then $(K \cap R, L \cap R) \in \mathcal{C}_{R}^{i_{0}}$.

Proof. Set $K_{1}=K \cap R$ and $L_{1}=L \cap R$. We note that $S^{-1} K_{1}=K$ and $S^{-1} L_{1}=L$. By Propositions 2.8 and 2.6, we get that

$$
\left.S^{-1} \text { (image } \theta_{K_{1}, L_{1}}^{i_{0}}(R)\right) \cong \text { image } \theta_{K, L}^{i_{0}}\left(S^{-1} R\right) .
$$

It follows that

$$
\sharp \operatorname{Ass}_{R}\left(\text { image } \theta_{K_{1}, L_{1}}^{i_{0}}(R)\right)=\infty \text {. }
$$

So $\left(K_{1}, L_{1}\right) \in \mathcal{C}_{R}^{i_{0}}$.

Proof of Lemma 2.10. (1) Suppose $\left(S^{-1} I, S^{-1} J\right) \preceq(K, L)$ for some $(K, L) \in$ $\mathcal{C}_{S^{-1} R}^{i_{0}}$. Then by Proposition 2.11, we get $(K \cap R, L \cap R) \in \mathcal{C}_{R}^{i_{0}}$. Notice that $(I, J) \preceq(K \cap R, L \cap R)$. By the maximality of $(I, J)$ in $\mathcal{C}_{R}^{i_{0}}$, we get that $I=K \cap R$ and $J=L \cap R$. So $\left(S^{-1} I, S^{-1} J\right)=(K, L)$.

(2) Set $K=S^{-1} I$ and $L=S^{-1} J$. Then by Proposition 2.11 and our hypotheses, $(K \cap R, L \cap R) \in \mathcal{C}_{R}^{i_{0}}$. Notice that $(I, J) \preceq(K \cap R, L \cap R)$. So by the maximality of $(I, J)$ in $\mathcal{C}_{R}^{i_{0}}$, we get $I=K \cap R$ and $J=L \cap R$.

2.12. Product of rings. Assume $R=R_{1} \times R_{2} \times \cdots \times R_{n}$. If $R$ is Noetherian then each $R_{i}$ is Noetherian. Note that an ideal $I$ in $R$ is of the form $I_{1} \times I_{2} \times \cdots \times I_{n}$, where $I_{j}$ is an ideal in $R_{j}$. Further note that $P$ is a prime in $R$ if and only if $P_{i}$ is a prime ideal in $R_{i}$ for some $i$ and $P_{j}=R_{j}$ for $j \neq i$. Thus $\operatorname{Spec}(R)$ is a disjoint union of $\operatorname{Spec}\left(R_{1}\right), \ldots, \operatorname{Spec}\left(R_{n}\right)$.

The following result is easy to verify.

Proposition 2.13. Let $M_{i}$ be $R_{i}$ modules. Then $M=M_{1} \times \cdots \times M_{n}$ is an $R$-module. Furthermore,

$$
\operatorname{Ass}_{R}(M)=\bigcup_{i=1}^{n} \operatorname{Ass}_{R_{i}}\left(M_{i}\right) .
$$

Remark 2.14. In the above proposition, a prime $P$ of $R_{i}$ is identified with the following prime of $R$ :

$$
R_{1} \times \cdots \times R_{i-1} \times P \times R_{i+1} \times \cdots \times R_{n} .
$$

The following result takes a little work. However, it is completely elementary and so we skip the proof.

Proposition 2.15. Assume the hypotheses of 2.12 hold. For each $i \geq 0$, we have an isomorphism

$$
H_{I}^{i}(R) \cong H_{I_{1}}^{i}\left(R_{1}\right) \times H_{I_{2}}^{i}\left(R_{2}\right) \times \cdots \times H_{I_{n}}^{i}\left(R_{n}\right)
$$


The following remark will be used often.

Remark 2.16. Let $R$ be a regular ring. Let $\left\{P_{1}, \ldots, P_{n}\right\}$ be minimal primes of $R$. Set $R_{i}=R / P_{i}$. Then

$$
R \cong R_{1} \times R_{2} \times \cdots \times R_{n}
$$

see [Matsumura 1989, Exercise 9.11]. Notice that the $R_{i}$ are regular domains.

If $R$ is excellent then each $R_{i}$ is excellent. Furthermore, if $\operatorname{dim} R=d$ then it is easy to see that $\operatorname{dim} R_{i} \leq d$ for all $i$ and $\operatorname{dim} R_{m}=d$ for some $m$.

By Propositions 2.15 and 2.13, it follows that if

$$
I=I_{1} \times \cdots \times I_{n}
$$

then Ass $H_{I}^{i}(R)$ is finite if and only if Ass $H_{I_{j}}^{i}\left(R_{j}\right)$ is finite for all $j$. Thus for the questions we are interested in, it suffices to assume that $R$ is a domain.

\section{The flat extension $R \rightarrow R[[X]]_{X}$}

In our arguments we need to assume that $R$ contains an uncountable field. When this is not the case, we consider the flat extension $R \rightarrow R[[X]]_{X}$. Set $S=R[[X]]$ and let $T=S_{X}=R[[X]]_{X}$, i.e., the ring obtained by inverting $X$.

Remark 3.1. (i) If $R$ contains a countable field $K$ then note that $K[[X]]$ is a subring of $S$ and so $K[[X]]_{X}$ is a subring of $T$. The field $K[[X]]_{X}$ is uncountable. Thus $T$ contains an uncountable field.

(ii) If $R$ is regular then so is $S$; see [Bruns and Herzog 1993, Theorem 2.2.13]. Therefore $T$ is also regular.

(iii) Let $R$ be an excellent regular ring of finite dimension containing a field of characteristic zero. As $S=R[[X]]$ is regular, it is universally catenary. Also $(X) \subseteq \operatorname{rad} S$ and $S /(X)=R$ is excellent. So by [Rotthaus 1980], we get that $S$ is excellent. It follows that $T$ is excellent.

The following proposition gives information about the behavior of primes when we pass from $R$ to $T$.

Proposition 3.2. Assume that the hypotheses above hold. Let $\mathfrak{p}$ be a prime in $R$. Then:

(i) $\mathfrak{p} T$ is a prime in $T$.

(ii) $T$ is a faithfully flat extension of $R$.

(iii) $\mathfrak{p} T \cap R=\mathfrak{p}$.

(iv) height $\mathfrak{p} T=$ height $\mathfrak{p}$.

(v) Let $P$ be a prime ideal in $T$. If $P \cap R=\mathfrak{p}$ then $P=\mathfrak{p} T$.

(vi) $\operatorname{dim} T=\operatorname{dim} R$. 
Proof. (i) Clearly $\mathfrak{p} S$ is a prime in $S$. Also $X \notin \mathfrak{p} S$. As $\mathfrak{p} T$ is localization of $\mathfrak{p} S$, we get that it is prime in $T$.

(ii) $T$ is clearly a flat $R$-algebra. Let $\mathfrak{m}$ be a maximal ideal of $R$. Then $\mathfrak{m} T$ is a prime ideal of $T$. In particular, $\mathfrak{m} T \neq T$. So $T$ is faithfully flat [Matsumura 1989, Theorem 7.2].

(iii) We have

$$
\mathfrak{p} T \cap R=\mathfrak{p} T \cap S \cap R=\mathfrak{p} S \cap R=\mathfrak{p} .
$$

(iv) By [loc. cit., Theorem 15.1], we get

$$
\text { height } \mathfrak{p} T=\text { height } \mathfrak{p}+\operatorname{dim} T_{\mathfrak{p} T} / \mathfrak{p} T_{\mathfrak{p} T} .
$$

As $\mathfrak{p} T_{\mathfrak{p} T}$ is the maximal ideal in $T_{\mathfrak{p} T}$, we get the required result.

(v) Again by [loc. cit., Theorem 15.1], we get

$$
\text { height } P=\text { height } \mathfrak{p}+\operatorname{dim} T_{P} / \mathfrak{p} T_{P} \text {. }
$$

Set $\kappa(\mathfrak{p})=R_{\mathfrak{p}} / \mathfrak{p} R_{\mathfrak{p}}$, the residue field of $R_{\mathfrak{p}}$. Then note that $T_{P} / \mathfrak{p} T_{P}$ is a localization of $\kappa(\mathfrak{p})[[X]]$ at a multiplicatively closed set containing $X$. It follows that $T_{P} / \mathfrak{p} T_{P}$ is a field. So height $P=$ height $\mathfrak{p}$. Now $P$ contains the prime ideal $\mathfrak{p} T$ which by (iii) also has height $=$ height $\mathfrak{p}$. So $P=\mathfrak{p} T$.

(vi) This follows easily from (iv) and (v).

We need Theorem 23.3 from [Matsumura 1989]. Unfortunately there is a typographical error in the statement of this theorem, so we state it here.

Theorem 3.3. Let $\varphi: A \rightarrow B$ be a homomorphism of Noetherian rings, and let $E$ be an A-module and $G$ a $B$-module. Suppose that $G$ is flat over $A$; then we have the following:

(i) If $\mathfrak{p} \in \operatorname{Spec} A$ and $G / \mathfrak{p} G \neq 0$ then

$$
\begin{aligned}
& { }^{a} \varphi\left(\operatorname{Ass}_{B}(G / \mathfrak{p} G)\right)=\operatorname{Ass}_{A}(G / \mathfrak{p} G)=\{\mathfrak{p}\} . \\
& \operatorname{Ass}_{B}\left(E \otimes_{A} G\right)=\bigcup_{\mathfrak{p} \in \operatorname{Ass}_{A}(E)} \operatorname{Ass}_{B}(G / \mathfrak{p} G) .
\end{aligned}
$$

Remark 3.4. In [Matsumura 1989], $\operatorname{Ass}_{A}(E \otimes G)$ is typed instead of $\operatorname{Ass}_{B}(E \otimes G)$. Also note that ${ }^{a} \varphi(P)=P \cap A$ for $P \in \operatorname{Spec} B$.

3.5. Let $M$ be an $R$-module. Set

$$
\operatorname{Ass}_{R}^{i}(M)=\left\{P \mid P \in \operatorname{Ass}_{R}(M) \text { and height } P=i\right\} .
$$

We now state the main result of this section. 
Theorem 3.6. Let $R$ be a Noetherian ring and let $M$ be an $R$-module. Set $S=$ $R[[X]]$ and $T=S_{X}$. Then:

(1) The mapping defined by

$$
\begin{aligned}
\psi: \operatorname{Ass}_{R}(M) & \rightarrow \operatorname{Ass}_{T}\left(M \otimes_{R} T\right), \\
\mathfrak{p} & \rightarrow \mathfrak{p} T,
\end{aligned}
$$

is a bijection.

(2) $\psi$ maps $\operatorname{Ass}_{R}^{i}(M)$ bijectively to $\operatorname{Ass}_{T}^{i}\left(M \otimes_{R} T\right)$.

Proof. (1) By Theorem 3.3, we get that

$$
\operatorname{Ass}_{T}\left(M \otimes_{R} T\right)=\bigcup_{\mathfrak{p} \in \operatorname{Ass}_{R}(M)} \operatorname{Ass}_{T}(T / \mathfrak{p} T) .
$$

By Proposition 3.2(i), we get that $\mathfrak{p} T$ is a prime ideal in $T$. So $\operatorname{Ass}_{T} T / \mathfrak{p} T=\{\mathfrak{p} T\}$. Thus

$$
\operatorname{Ass}_{T}\left(M \otimes_{R} T\right)=\left\{\mathfrak{p} T \mid \mathfrak{p} \in \operatorname{Ass}_{R}(M)\right\} .
$$

So the map $\psi$ is well-defined and surjective. By Proposition 3.2(iii), we get that it is injective.

(2) This follows from (1) and Proposition 3.2(iv).

An immediate corollary is this:

Corollary 3.7. Assume that the hypotheses of Theorem 3.6 hold. Then:

(1) $\operatorname{Ass}_{R} M$ is an infinite set if and only if $\operatorname{Ass}_{T} M \otimes_{R} T$ is an infinite set.

(2) $\mathrm{Ass}_{R}^{i} M$ is an infinite set if and only if $\mathrm{Ass}_{T}^{i} M \otimes_{R} T$ is an infinite set.

\section{Countable prime avoidance}

4.1. Setup. In this section, $R$ is a Noetherian ring containing an uncountable field $K$. We describe a construction which we will use often. The essential ingredient is the following well-known countable avoidance. We give a proof due to lack of a suitable reference.

Lemma 4.2. Assume the hypotheses of 4.1 hold. Let $\left\{I_{n}\right\}_{n \geq 1}$ be ideals in $R$ and let $J$ be another ideal in $R$. If $J \subseteq \bigcup_{n \geq 1} I_{n}$ then $J \subseteq I_{m}$ for some $m$.

Proof. Let $J=\left(x_{1}, \ldots, x_{c}\right)$. Let $V=K x_{1}+K x_{2}+\cdots+K x_{c}$. Then $V$ is a finite dimensional $K$-vector space. Also, clearly $V=\bigcup_{n \geq 1} V \cap I_{n}$. As $K$ is an uncountable field, we get that $V \cap I_{m}=V$ for some $m$. Thus $x_{i} \in I_{m}$ for all $i$. So $J \subseteq I_{m}$. 
4.3. Construction. Assume that the hypotheses of 4.1 hold and that $\left\{\mathfrak{p}_{n}\right\}_{n \geq 1}$ is a sequence of primes in $R$. Also assume that $\mathfrak{p}_{i} \not \subseteq \mathfrak{p}_{j}$ for $i \neq j$. Consider the multiplicatively closed set

$$
S=R \backslash \bigcup_{n \geq 1} \mathfrak{p}_{n}
$$

Set $T=S^{-1} R$. The following result gives information about primes in $T$.

Proposition 4.4. Assume the hypotheses of 4.3 hold. We have:

(1) If $\mathfrak{p} T$ is a prime in $T$, where $\mathfrak{p}$ is a prime in $R$, then $\mathfrak{p} \subseteq \mathfrak{p}_{n}$ for some $n$.

(2) $\mathfrak{p}_{i} T \nsubseteq \mathfrak{p}_{j} T$ for $i \neq j$.

(3) $\mathfrak{p}_{n} T$ are distinct maximal ideals of $T$.

(4) $\mathrm{m}-\operatorname{Spec}(T)=\left\{\mathfrak{p}_{n} T\right\}_{n \geq 1}$.

Proof. (1) We have $\mathfrak{p} \cap S=\varnothing$. So $\mathfrak{p} \subseteq \bigcup_{n \geq 1} \mathfrak{p}_{n}$. By Lemma 4.2, we get that $\mathfrak{p} \subseteq \mathfrak{p}_{n}$ for some $n$.

(2) If $\mathfrak{p}_{i} T \subseteq \mathfrak{p}_{j} T$ for some $i \neq j$, then intersecting with $R$, we get $\mathfrak{p}_{i} \subseteq \mathfrak{p}_{j}$ for some $i \neq j$; a contradiction.

(3) Let $\mathfrak{p}_{n} T \subseteq P$ for some prime $P$ of $T$. Say $P=\mathfrak{p} T$, where $\mathfrak{p}$ is a prime in $R$. By (1) we get that $\mathfrak{p} T \subseteq \mathfrak{p}_{m} T$ for some $m$. By (2) we get $m=n$. So $P=\mathfrak{p}_{n} T$. Thus $\mathfrak{p}_{n} T$ is a maximal ideal in $T$. That they are all distinct follows from (2).

(4) This follows from (1) and (3).

We will need the following intersection result:

Proposition 4.5. Assume that the hypotheses of 4.3 hold. Let $\Lambda$ be any subset of $\left\{\mathfrak{p}_{n}\right\}_{n \geq 1}$ (possibly infinite). Set

$$
U=\bigcap_{\mathfrak{p} \in \Lambda} \mathfrak{p} \text { and } \quad V=\bigcap_{\mathfrak{p} \in \Lambda} \mathfrak{p} T .
$$

Then $U T=V$.

Proof. Clearly $U T \subseteq V$ Let $\xi=a / s \in V$ and $\mathfrak{p} \in \Lambda$. As $\xi \in \mathfrak{p} T$, we get that $\xi=r / s_{1}$, where $r \in \mathfrak{p}$. It follows that there exists $s^{\prime} \in S$ such that $s^{\prime} s_{1} a \in \mathfrak{p}$. As $s^{\prime} s_{1} \notin \mathfrak{p}$, we get that $a \in \mathfrak{p}$. Thus $a \in U$. Therefore $\xi \in U T$, and hence $V=U T$.

\section{Proof of Theorem 1.4}

Proof of Theorem 1.4. Suppose $\mathcal{C}_{R}^{i_{0}}$ is nonempty for some $i_{0}$. Let $(I, J)$ be a maximal element in $\mathcal{C}_{R}^{i_{0}}$. It follows from Proposition 2.1 that $\operatorname{Ass}_{R}\left(\right.$ image $\theta_{I, J}^{i_{0}}$ ) is a countably infinite set. As $\operatorname{dim} R$ is finite, we can choose an infinite subset $\left\{\mathfrak{p}_{n}\right\}_{n \geq 1}$ 
of $\operatorname{Ass}_{R}$ (image $\theta_{I, J}^{i_{0}}$ ) such that height $\mathfrak{p}_{r}=$ height $\mathfrak{p}_{s}$ for all $r, s$. Clearly the primes $\left\{\mathfrak{p}_{n}\right\}_{n \geq 1}$ are mutually incomparable. Set

$$
S=R \backslash \bigcup_{n \geq 1} \mathfrak{p}_{n} \quad \text { and } \quad A=S^{-1} R .
$$

By Proposition 4.4, we get m-Spec $(A)=\left\{\mathfrak{p}_{n} A\right\}_{n \geq 1}$. Furthermore, by construction, height $\mathfrak{p}_{n} A=$ height $\mathfrak{p}_{n}$ is constant. Also by Lemma 2.8 , we get that

$$
\operatorname{Ass}_{A}\left(\text { image } \theta_{I A, J A}^{i_{0}}\right) \supseteq \mathrm{m}-\operatorname{Spec}(A) .
$$

By Lemma 2.10, we also get that $(I A, J A)$ is a maximal element of $\mathcal{C}_{A}^{i_{0}}$.

Suppose, if possible, that $g=$ height $I A<i_{0}$.

Claim 1: $\operatorname{Ass}_{A} H_{I A}^{i_{0}}(A)$ is an infinite set.

Suppose, if possible, that $\operatorname{Ass}_{A} H_{I A}^{i_{0}}(A)=\left\{Q_{1}, Q_{2}, \ldots, Q_{s}\right\}$ is a finite set. Notice that height $Q_{i} \geq i_{0}>g$ for all $i$. Note that $I A$ is a radical ideal. Let $I A=P_{1} \cap P_{2} \cap \cdots \cap P_{r}$ for some primes $P_{j}$. Say height $P_{1}=g$. Choose $x_{i} \in Q_{i} \backslash P_{1}$. Set $x=x_{1} x_{2} \cdots x_{s}$. Then $x \in Q_{i}$ for all $i$. Also $x \notin P_{1}$, so $x \notin I A$.

We have an exact sequence

$$
\cdots \rightarrow H_{I A+(x)}^{i_{0}}(A) \stackrel{\theta_{I A+(x), I A}^{i_{0}}}{\longrightarrow} H_{I A}^{i_{0}}(A) \rightarrow\left(H_{I A}^{i_{0}}(A)\right)_{x} \rightarrow \cdots .
$$

As $x \in Q_{i}$ for all $i$, we get that $\left(H_{I A}^{i_{0}}(A)\right)_{x}=0$. Thus $\theta_{I A+(x), I A}^{i_{0}}$ is surjective. Notice that

$$
\theta_{I A+(x), J A}^{i_{0}}=\theta_{I A, J A}^{i_{0}} \circ \theta_{I A+(x), I A}^{i_{0}} .
$$

As $\theta_{I A+(x), I A}^{i_{0}}$ is surjective, it follows that $\operatorname{Ass}_{A}$ (image $\left.\theta_{I A+(x), J A}^{i_{0}}\right)$ is an infinite set. So

$$
(I A+(x), J A) \in \mathcal{C}_{A}^{i_{0}} .
$$

Also $x \notin I A$. This contradicts the maximality of $(I A, J A)$ in $\mathcal{C}_{A}^{i_{0}}$. Thus $\operatorname{Ass}_{A} H_{I A}^{i_{0}}(A)$ is an infinite set.

Now let $\Lambda$ be an infinite subset of $\operatorname{Ass}_{A}\left(H_{I A}^{i_{0}}(A)\right)$. Set

Clearly $I A \subseteq W_{\Lambda}$.

$$
W_{\Lambda}=\bigcap_{P \in \Lambda} P \text {. }
$$

Claim 2: $I A=W_{\Lambda}$.

Suppose, if possible, that there exists $x \in W_{\Lambda} \backslash I A$. We have an exact sequence

$$
\cdots \rightarrow H_{I A+(x)}^{i_{0}}(A) \stackrel{\theta_{I A+(x), I A}^{i_{0}}}{\longrightarrow} H_{I A}^{i_{0}}(A) \stackrel{\pi_{i_{0}}}{\longrightarrow}\left(H_{I A}^{i_{0}}(A)\right)_{x} \rightarrow \cdots .
$$

For $P \in \Lambda$, let $P=\left(0: a_{P}\right)$ for some $a_{P} \in H_{I A}^{i_{0}}(A)$. Clearly $\pi_{i_{0}}\left(a_{P}\right)=0$ for all $P \in \Lambda$. It follows that

$$
\Lambda \subseteq \operatorname{Ass}_{A}\left(\operatorname{image}\left(\theta_{I A+(x), I A}^{i_{0}}\right)\right)
$$


Thus $(I A+(x), I A) \in \mathcal{C}_{A}^{i_{0}}$. Also $x \notin I A$. This contradicts the maximality of $(I A, J A)$ in $\mathcal{C}_{A}^{i_{0}}$. Thus Claim 2 is true.

Let

$$
L_{j}=\left\{P \mid P \in \operatorname{Ass}_{A}\left(H_{I A}^{i_{0}}(A)\right) \text { and height } P=j\right\} .
$$

As $\operatorname{dim} A$ is finite, we get by Claim 1 that $L_{j}$ is infinite for some $j$. Choose $j_{0}$ to be the maximum $j$ with $L_{j}$ infinite. Set

$$
\Lambda=L_{j_{0}} \backslash\left\{\mathfrak{p} \mid \mathfrak{p} \text { a minimal prime of } I A \text { and height } \mathfrak{p}=j_{0}\right\} .
$$

Clearly $\Lambda$ is an infinite set. Set

$$
T=A \backslash \bigcup_{P \in \Lambda} P \quad \text { and } \quad B=T^{-1} A .
$$

By Proposition 4.4, we get that

$$
\mathrm{m}-\operatorname{Spec}(B)=\{P B \mid P \in \Lambda\} .
$$

It follows from Claim 2 and Proposition 4.5 that

$$
I B=\bigcap_{P \in \Lambda} P B .
$$

Thus $I B$ is the Jacobson radical of $B$. Note we also get that $\operatorname{Ass}_{B} H_{I B}^{i_{0}}(B)$ contains $\mathrm{m}-\operatorname{Spec}(B)$.

Since $R$ is excellent, $A$ is excellent and hence $B$ is excellent. Therefore $\operatorname{Reg}(B / I B)$ is an open set. As $I B$ is a radical ideal, we get that $\operatorname{Reg}(B / I B)$ is nonempty. Since $\operatorname{rad}(B / I B)=0$, it follows that there exists a maximal ideal $\mathfrak{m}$ of $B$ with $(B / I B)_{\mathfrak{m}}$ a regular local ring. As $B$ is a regular ring, $B_{\mathfrak{m}}$ is a regular ring. Also note that by our construction, $I B_{\mathfrak{m}} \neq \mathfrak{m} B_{\mathfrak{m}}$. As $B_{\mathfrak{m}} / I B_{\mathfrak{m}}$ is regular, we get that $I B_{\mathfrak{m}}$ is generated by part of a regular system of parameters. Say $I B_{\mathfrak{m}}=\left(x_{1}, \ldots, x_{c}\right)$ and $c<\operatorname{dim} B_{\mathfrak{m}}$. We now note that

$$
H_{I B_{\mathfrak{m}}}^{j}\left(B_{\mathfrak{m}}\right)=0 \text { for } j \neq c \quad \text { and } \quad \text { Ass } H_{I B_{\mathfrak{m}}}^{c}\left(B_{\mathfrak{m}}\right)=\left\{\left(x_{1}, \ldots, x_{c}\right)\right\} \text {. }
$$

It follows that $\mathfrak{m} \notin \operatorname{Ass}_{B} H_{I B}^{i_{0}}(B)$; a contradiction. Thus height $I A=i_{0}$.

Let $I A=P_{1} \cap P_{2} \cap \cdots \cap P_{s} \cap Q_{1} \cap Q_{2} \cap \cdots \cap Q_{l}$, where height $P_{j}=i_{0}$ for all $j$ and height $Q_{j} \geq i_{0}+1$ for all $j$. Set $K=P_{1} \cap \cdots \cap P_{s}$. It is well known that the natural map

$$
\theta_{K, I A}^{i_{0}}: H_{K}^{i_{0}}(A) \rightarrow H_{I A}^{i_{0}}(A) \quad \text { is an isomorphism. }
$$

It follows that $(K, J A) \in \mathcal{C}_{A}^{i_{0}}$. By the maximality of $(I A, J A)$ in $\mathcal{C}_{A}^{i_{0}}$, we get that $K=I A$.

Now assume $\operatorname{Ass}_{R}\left(H_{L}^{r}(R)\right)$ is a finite set for all $r<i_{0}$ and for all ideals $L$ of $R$. In particular, $\operatorname{Ass}_{R}\left(H_{J}^{i_{0}-1}(R)\right)$ is a finite set. So $\operatorname{Ass}_{A}\left(H_{J A}^{i_{0}-1}(A)\right)$ is a finite set. Suppose, if possible, that height $J A<i_{0}-1$. Let

$$
\operatorname{Ass}_{A}\left(H_{J A}^{i_{0}-1}(A)\right)=\left\{Q_{1}, Q_{2}, \ldots, Q_{s}\right\} .
$$


Notice height $Q_{j} \geq i_{0}-1$ for all $j$. Let $J A=P_{1} \cap P_{2} \cdots \cap P_{r}$, where height $P_{1}=$ height $J A<i_{0}-1$. Choose $y_{j} \in Q_{j} \backslash P_{1}$. Also choose $x \in I \backslash P_{1}$. Set $t=$ $y_{1} y_{2} \cdots y_{s} x$. Then $t \in Q_{j}$ for all $j$. We note that

$$
\left(H_{J A}^{i_{0}-1}(A)\right)_{t}=0 .
$$

Also note that as $t \notin P_{1}$, we get $t \notin J A$.

Thus we have a commutative diagram

0

$$
H_{I A+(t)}^{i_{0}}
$$

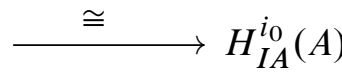
$\theta_{I A, J A}^{i_{0}}$

0

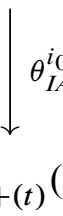
$H_{J A+(t)}^{i_{0}}(A)$

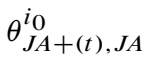

We note that as $t \in I$, we get $t \in \operatorname{rad}(A)$. Let $\mathfrak{m}$ be a maximal ideal in $A$ and let $\left(0: a_{\mathfrak{m}}\right)=\mathfrak{m}$, where $a_{\mathfrak{m}} \in$ image $\theta_{I A, J A}^{i_{0}}$. Clearly $\pi\left(a_{\mathfrak{m}}\right)=0$. Thus it follows that $\mathfrak{m} \in \operatorname{Ass}_{A}\left(H_{J A+(t)}^{i_{0}}(A)\right)$. A simple diagram chase shows that, in fact,

$$
\mathfrak{m} \in \operatorname{Ass}_{A}\left(\text { image } \theta_{I A, J A+(t)}^{i_{0}}\right) .
$$

Thus $(I A, J A+(t)) \in \mathcal{C}_{A}^{i_{0}}$. This contradicts the maximality of $(I A, J A)$ in $\mathcal{C}_{A}^{i_{0}}$. Therefore height $J A=i_{0}-1$.

As an immediate consequence, we get this:

Proof of Theorem 1.5. (i) $\Longrightarrow$ (ii): This is obvious.

(ii) $\Longrightarrow$ (i): By Theorem 1.4, it follows that Lyubeznik's conjecture holds for all excellent regular domains of dimension $\leq d$ containing an uncountable field of characteristic zero. By results in Section 3, it follows that Lyubeznik's conjecture holds for all excellent regular domains of dimension $\leq d$ containing a field of characteristic zero. By Remark 2.16, the result holds for all excellent regular rings of dimension $\leq d$ containing a field of characteristic zero.

\section{Proof of Theorem 1.3}

In this section we prove Theorem 1.3. We will need the following result (see [Matsumura 1989, proof of Theorem 31.1]).

Lemma 6.1. Let $\left\{Q_{n}\right\}_{n \geq 1}$ be an infinite family of primes in a Noetherian ring $T$, and let $P$ be another prime ideal in $T$ with $P \subseteq Q_{n}$ for all $n$. Suppose that height $\left(Q_{n} / P\right)=1$ for all $n$. Then

$$
\bigcap_{n \geq 1} Q_{n}=P
$$


We also need Corollary 7.8. As the techniques to prove it are totally different, we postpone the proof of Corollary 7.8 to the next section.

Proof of Theorem 1.3. By Remark 2.16, we may assume that $R$ is a domain. By results in Section 3, we may assume that $R$ contains an uncountable field of characteristic zero.

Suppose, if possible, that for some ideal $K$ of $R$, we have $\operatorname{Ass}_{R}\left(H_{K}^{d-1}(R)\right)$ is an infinite set. So $(K, K) \in \mathcal{C}_{R}^{d-1}$. Thus $\mathcal{C}_{R}^{d-1} \neq \varnothing$. Let $(I, J)$ be a maximal element in $\mathcal{C}_{R}^{d-1}$. We now do the construction as in Theorem 1.4. Then in the ring $A=S^{-1} R$, we have

(a) $(I A, J A)$ is a maximal element in $\mathcal{C}_{A}^{d-1}$,

(b) height $I A=d-1$,

(c) $I A=P_{1} \cap P_{2} \cap \cdots \cap P_{r}$, where $P_{i}$ is a prime in $A$ of height $d-1$,

(d) $\operatorname{Ass}_{A}$ (image $\left.\theta_{I A, J A}^{d-1}\right) \supseteq \mathrm{m}-\operatorname{Spec}(A)$,

(e) $\mathrm{m}-\operatorname{Spec}(A)$ is a countably infinite set.

We now note that $\operatorname{dim} A=d$. This is so since for any Noetherian ring $T$ of dimension $n$ and an ideal $L$ of $T$, we have $\operatorname{Ass}_{T}\left(H_{L}^{n}(T)\right)$ is a finite set. Thus $\operatorname{dim} A \neq d-1$. Furthermore, by Grothendieck's vanishing theorem, it is not possible that $\operatorname{dim} A<d-1$. Thus $\operatorname{dim} A=d$. Again by Theorem 1.4, we get that height $\mathfrak{m}=d$ for all maximal ideals of $A$. We note that $I A \subseteq \operatorname{rad}(A)$.

Claim 1: There exists a localization $B$ of $A$ such that

(1) $\operatorname{dim} B=d$,

(2) height $\mathfrak{m}=d$ for all maximal ideals $\mathfrak{m}$ of $B$,

(3) $\mathrm{m}-\mathrm{Spec}(B)$ is a countably infinite set,

(4) $\operatorname{Ass}_{B}\left(\right.$ image $\left.\theta_{I B, J B}^{d-1}\right) \supseteq \mathrm{m}-\operatorname{Spec}(B)$,

(5) $I B$ is a prime ideal of height $d-1$,

(6) $I B=\operatorname{rad}(B)$.

To prove the claim, we recall that $I A=P_{1} \cap P_{2} \cap \cdots \cap P_{r}$, where $P_{i}$ is a prime ideal in $A$ of height $d-1$. We consider two cases.

Case 1: $r=1$. Then $I A=P_{1}$ is a prime ideal of height $d-1$. Also $P_{1} \subseteq \mathfrak{m}$ for all maximal ideals $\mathfrak{m}$ of $A$. As height $\mathfrak{m}=d$ for all maximal ideals of $A$ and as $\mathrm{m}-\operatorname{Spec}(A)$ is a countably infinite set, by Lemma 6.1 we get $I A=P_{1}=\operatorname{rad}(A)$. Thus we can take $B$ to be $A$.

Case 2: $r \geq 2$. Consider the sets

$$
Y_{i}=\left\{\mathfrak{m} \mid \mathfrak{m} \in \mathrm{m}-\operatorname{Spec}(A) \text { and } \mathfrak{m} \supseteq P_{i}\right\} .
$$


As $I A \subseteq \operatorname{rad}(A)$, we get $Y_{1} \cup Y_{2} \cup \cdots \cup Y_{r}=\mathrm{m}-\operatorname{Spec}(A)$. So there exists $i$ such that $Y_{i}$ is an infinite set. After relabeling, we may assume $i=1$. Set

$$
T_{1}=A \backslash \bigcup_{\mathfrak{m} \in Y_{1}} \mathfrak{m} \quad \text { and } \quad A_{1}=T_{1}^{-1} A .
$$

Let $I A_{1}=Q_{1} \cap Q_{2} \cap \cdots \cap Q_{s}$ be an irredundant primary decomposition of $I A_{1}$ with $Q_{1}=P_{1} A_{1}$. (Note as $I A_{1}$ is a radical ideal, all $Q_{i}$ are prime ideals.) We note that height $Q_{1}=d-1$. Furthermore, height $\mathfrak{n}=d$ for each maximal ideal of $A_{1}$. Also by Proposition 4.4, $\sharp \mathrm{m}-\operatorname{Spec}\left(A_{1}\right)=\sharp Y_{1}=\infty$. As $Q_{1} \subseteq \mathfrak{m}$ for each maximal ideal of $A_{1}$, by Lemma 6.1 we get $Q_{1}=\operatorname{rad}\left(A_{1}\right)$.

We also note that $\operatorname{Ass}_{A_{1}}$ (image $\left.\theta_{I A_{1}, J A_{1}}^{d-1}\right) \supseteq \mathrm{m}-\operatorname{Spec}\left(A_{1}\right)$ and $I A_{1} \subseteq \operatorname{rad}\left(A_{1}\right)$. For $j \geq 2$, consider the sets

$$
Y_{j}^{\prime}=\left\{\mathfrak{m} \mid \mathfrak{m} \in \mathrm{m}-\operatorname{Spec}\left(A_{1}\right) \text { and } \mathfrak{m} \supseteq Q_{j}\right\} .
$$

Claim 2: $Y_{j}^{\prime}$ is a finite set for all $j \geq 2$.

Suppose, if possible, that $Y_{j}$ is an infinite set for some $j$. Then by Lemma 6.1, we get

$$
Q_{j}=\bigcap_{\mathfrak{m} \in Y_{j}^{\prime}} \mathfrak{m} \supseteq \operatorname{rad}\left(A_{1}\right)=Q_{1} .
$$

This contradicts the fact that $Q_{1} \cap \cdots \cap Q_{s}$ is an irredundant primary decomposition of $I A_{1}$. Thus Claim 2 is proved.

Now set

$$
\Lambda=\mathrm{m}-\operatorname{Spec}\left(A_{1}\right) \backslash \bigcup_{j \geq 2} Y_{j}^{\prime}, \quad T_{2}=A_{1} \backslash \bigcup_{\mathfrak{m} \in \Lambda} \mathfrak{m} \quad \text { and } \quad B=T_{2}^{-1} A_{1} .
$$

It is easy to prove that $B$ satisfies all the assertions in Claim 1 .

We now note that $B$ is excellent. So $\operatorname{Reg}(B / I B)$ is an open set. As $I B$ is a prime ideal, we get that $\operatorname{Reg}(B / I B)$ is nonempty. Since $\operatorname{rad}(B / I B)=0$, it follows that there exists a maximal ideal $\mathfrak{m}$ of $B$ with $(B / I B)_{\mathfrak{m}}$ a regular local ring. As $B$ is a regular ring, $B_{\mathfrak{m}}$ is a regular ring. Also note that height $I B_{\mathfrak{m}}=d-1$ and height $\mathfrak{m} B_{\mathfrak{m}}=d$. As $B_{\mathfrak{m}} / I B_{\mathfrak{m}}$ is regular, we get that $I B_{\mathfrak{m}}$ is generated by part of a regular system of parameters. Say $I B_{\mathfrak{m}}=\left(x_{1}, \ldots, x_{d-1}\right)$ and say $\mathfrak{m} B_{\mathfrak{m}}=$ $\left(x_{1}, \ldots, x_{d-1}, x_{d}\right)$. Let $k$ be the residue field of $B_{\mathfrak{m}}$. Set $C=\widehat{B_{\mathfrak{m}}}$. Note that $C=$ $k\left[\left[x_{1}, \ldots, x_{d}\right]\right]$ and $I C=\left(x_{1}, \ldots, x_{d-1}\right)$. Let $\mathfrak{n}=\left(x_{1}, \ldots, x_{d}\right) C$ be the maximal ideal of $C$. By our assumption, $\mathfrak{n} \in \operatorname{Ass}_{C}$ (image $\theta_{I C, J C}^{d-1}$ ). However, this contradicts Corollary 7.8. Thus $\operatorname{Ass}_{R}\left(H_{K}^{d-1}(R)\right)$ is a finite set for all ideals $K$ of $R$.

\section{Simplicity of a local cohomology module}

The references for this section are [Björk 1979; Lyubeznik 1993]. Let $\mathcal{O}=$ $K\left[\left[X_{1}, \ldots, X_{n}\right]\right]$, where $K$ is a field of characteristic zero. Let $D$ be the ring 
of $K$-linear differential operators on $D$. By the work of Lyubeznik, it is known that if $I$ is an ideal in $\mathcal{O}$ then $H_{I}^{i}(\mathcal{O})$ are finitely generated $D$-modules for all $i \geq 0$. The main goal of this section is to prove that if $P_{g}=\left(X_{1}, \ldots, X_{g}\right)$ for $1 \leq g \leq n$ then $H_{P_{g}}^{g}(\mathcal{O})$ is simple as a $D$-module. This result is known in the polynomial case and is easy to prove. A few experts we asked also claimed that this fact is known even in the case of power series rings. However, none of them had a reference for this result. As this result is crucial for us; we give a proof of this fact.

7.1. Consider the $\sum$-filtration where $\sum_{k}=\left\{Q \in D \mid Q=\sum_{|\alpha| \leq k} q_{\alpha}(\boldsymbol{X}) \partial^{\alpha}\right\}$ is the set of differential operators of order $\leq k$. The associated graded ring gr $D=\sum_{0} \oplus \sum_{1} / \sum_{0} \oplus \cdots$ is isomorphic to the polynomial ring $\mathcal{O}\left[\zeta_{1}, \ldots, \zeta_{n}\right]$, where $\zeta_{i}$ is the image of $\partial_{i}$ in $\sum_{1} / \sum_{0}$.

7.2. Let $M$ be a finitely generated $D$-module. We consider filtrations $\mathcal{F}$ of $K$-linear subspaces of $M$ with the property that $\mathcal{F}_{i}=0$ for $i<0, \mathcal{F}_{i} \subseteq \mathcal{F}_{i+1}, M=\bigcup_{i \geq 0} \mathcal{F}_{i}$ and $\sum_{i} \mathcal{F}_{j} \subseteq \mathcal{F}_{i+j}$. Note that $\operatorname{gr}_{\mathcal{F}} M=\bigoplus_{i \geq 0} \mathcal{F}_{i} / \mathcal{F}_{i-1}$ is a gr $D$-module. We say $\mathcal{F}$ is a good filtration of $M$ if $\operatorname{gr}_{\mathcal{F}} M$ is finitely generated as a gr $D$-module. We note that every finitely generated $D$-module $M$ has a good filtration.

7.3. Let $\mathcal{M}$ be the unique graded maximal ideal of gr $D$. Let $\mathcal{F}$ be a good filtration on $M$. Then note that $\operatorname{dim} \operatorname{gr}_{\mathcal{F}} M=\operatorname{dim}\left(\operatorname{gr}_{\mathcal{F}} M\right)_{\mathcal{M}}$; see [Bruns and Herzog 1993, Theorem 1.5.8]. Let $e\left(\mathrm{gr}_{\mathcal{F}} M\right)$ be the multiplicity of $\left(\mathrm{gr}_{\mathcal{F}} M\right)_{\mathcal{M}}$ with respect to the maximal ideal $\mathcal{M}(\operatorname{gr} D)_{\mathcal{M}}$ of the regular local ring $(\operatorname{gr} D)_{\mathcal{M}}$. Let $\mathcal{F}, \mathcal{G}$ be two good filtrations on $M$. Then by [Björk 1979, Lemma 6.2, Chapter 2], we get that

$$
\operatorname{dim} \operatorname{gr}_{\mathcal{F}} M=\operatorname{dim} \operatorname{gr}_{\mathcal{G}} M \text { and } e\left(\operatorname{gr}_{\mathcal{F}} M\right)=e\left(\operatorname{gr}_{\mathcal{G}} M\right) .
$$

Thus if $\mathcal{F}$ is a good filtration on $M$ then we can set

$$
\operatorname{dim} M=\operatorname{dim} \operatorname{gr}_{\mathcal{F}} M \quad \text { and } \quad e_{\mathcal{M}}(M)=e\left(\operatorname{gr}_{\mathcal{F}} M\right) .
$$

7.4. It is well known that if $M$ is a nonzero $D$-module then $\operatorname{dim} M \geq n$. If $\operatorname{dim} M=n$ or if $M=0$ then we say $M$ is a holonomic $D$-module. By the work of Lyubeznik, it is known that if $I$ is an ideal in $\mathcal{O}$ then $H_{I}^{i}(\mathcal{O})$ is a holonomic $D$-module for all $i \geq 0$.

We need the following lemma and its corollary:

Lemma 7.5. Let $M$ be a holonomic $D$-module. If $M \neq 0$ and $M$ is not simple then $e_{\mathcal{M}}(M) \geq 2$.

Proof. As $M$ is not simple, it has a proper nonzero submodule $K$. Set $C=M / K$. Then $K, C$ are nonzero holonomic $D$-modules.

Let $\mathcal{F}$ be a good filtration on $M$. Set $\overline{\mathcal{F}}=$ quotient filtration on $C$ and let $\mathcal{G}=\left\{\mathcal{F}_{n} \cap K\right\}_{n \geq 0}$ be the induced filtration on $K$. Then we have an exact sequence 
of graded gr $D$-modules,

$$
0 \rightarrow \mathrm{gr}_{\mathcal{G}} K \rightarrow \mathrm{gr}_{\mathcal{F}} M \rightarrow \mathrm{gr}_{\overline{\mathcal{F}}} C \rightarrow 0 .
$$

Thus $\operatorname{gr}_{\mathcal{G}} K$ and $\operatorname{gr}_{\overline{\mathcal{F}}} C$ are finitely generated gr $D$ modules. So $\overline{\mathcal{F}}$ is a good filtration of $C$ and $\mathcal{G}$ is a good filtration of $K$.

All the modules in equation $(*)$ have dimension $n$. Computing multiplicities we get

$$
e_{\mathcal{M}}(M)=e_{\mathcal{M}}(K)+e_{\mathcal{M}}(C) \geq 2
$$

As an immediate corollary, we obtain this:

Corollary 7.6. Let $M$ be a holonomic $D$-module. If $e_{\mathcal{M}}(M)=1$ then $M$ is a simple D-module.

The main result of this section is this:

Lemma 7.7. Let $\mathcal{O}=K\left[\left[X_{1}, \ldots, X_{n}\right]\right]$ and let $P_{g}=\left(X_{1}, \ldots, X_{g}\right)$ for $g \geq 1$. Then $e_{\mathcal{M}}\left(H_{P_{g}}^{g}(\mathcal{O})\right)=1$. Thus $H_{P_{g}}^{g}(\mathcal{O})$ is a simple D-module.

Proof. We first consider the case when $g=n$. So $P_{n}=\mathfrak{m}=\left(X_{1}, \ldots, X_{n}\right)$. In this case it is well known that $H_{\mathfrak{m}}^{n}(\mathcal{O}) \cong D / D \mathfrak{m}=K\left[\overline{\partial_{1}}, \ldots, \overline{\partial_{n}}\right]$ as $D$-modules. Let $\mathcal{F}_{i}=\left\{\sum_{|\alpha| \leq i} a_{\alpha} \bar{\partial}^{\alpha} \mid a_{\alpha} \in K\right\}$. Let $Q \in \sum_{i}$ be a differential operator of order $\leq i$. Then notice that $Q$ can also be written as $Q=\sum_{|\alpha| \leq i} \partial^{\alpha} a_{\alpha}$. Set $a_{\alpha}=c_{\alpha}+t_{\alpha}$, where $a_{\alpha} \in K$ and $t_{\alpha} \in \mathfrak{m}$. Then notice that the image of $Q$ in $D / D \mathfrak{m}$ is $\sum_{|\alpha| \leq i} c_{\alpha} \bar{\partial}^{\alpha}$. Thus $\mathcal{F}$ is the quotient filtration of $\sum$. Therefore $\mathcal{F}$ is a good filtration on $D / D \mathfrak{m}$. Also note that

$$
\operatorname{gr}_{\mathcal{F}} D / D \mathfrak{m}=\operatorname{gr} D / \mathfrak{m} \operatorname{gr} D=K\left[\zeta_{1}, \ldots, \zeta_{n}\right] .
$$

Clearly $e_{\mathcal{M}}(D / D \mathfrak{m})=1$. Also note that $X_{i} \mathcal{F}_{v} \subseteq \mathcal{F}_{\nu-1}$ for all $v \geq 0$.

We now consider the case when $g<n$. Set $S=K\left[\left[X_{1}, \ldots, X_{g}\right]\right]$ and $\mathfrak{n}=$ $\left(X_{1}, \ldots, X_{g}\right)$. Let $D^{\prime}$ be the ring of $K$-linear differential operators on $S$. Let $M=H_{\mathfrak{n}}^{g}(S)=D^{\prime} / D^{\prime} \mathfrak{n}$. Set $N=H_{P_{g}}^{g}(\mathcal{O})=M \otimes_{S} \mathcal{O}$. We note that the $D$-module structure on $N$ is given by $\partial_{1}, \ldots, \partial_{g}$ acting on $M$ and $\partial_{i}$ acting on $\mathcal{O}$ for $i>g$. Also note that for $r \in \mathcal{O}, m \in M$ and $t \in \mathcal{O}$, we have $r \cdot(m \otimes t)=m \otimes r t$. Let $\mathcal{F}$ be the $D^{\prime}$-filtration on $M$ as discussed earlier. Set

$$
\Omega_{v}=\left\{\sum_{\text {finite sum }} m_{\alpha} \otimes r_{\alpha} \mid m_{\alpha} \in \mathcal{F}_{\nu} \text { and } r_{\alpha} \in \mathcal{O}\right\} \text {. }
$$

Let $\xi=a \partial_{1}^{a_{1}} \cdots \partial_{g}^{a_{g}} \cdots \partial_{n}^{a_{n}} \in \sum_{i}$. So $\sum_{k=1}^{n} a_{k} \leq i$. In particular, $\sum_{k=1}^{g} a_{k} \leq i$. Let $m \otimes r \in \Omega_{v}$. Then

$$
\xi \cdot(m \otimes r)=\left(a\left(\partial_{1}^{a_{1}} \cdots \partial_{g}^{a_{g}} m\right)\right) \otimes\left(a\left(\partial_{g+1}^{a_{g+1}} \cdots \partial_{n}^{a_{n}} r\right)\right) \in \Omega_{v+i} .
$$


Thus $\Omega$ is a filtration on $N$ compatible with the $\sum$-filtration on $D$. Therefore $\operatorname{gr}_{\Omega} N$ is a gr $D$-module.

We first assert that $\operatorname{gr}_{\Omega} N$ is generated by $\overline{1 \otimes 1} \in \Omega_{0}$. Let $\xi=m \otimes r \in \Omega_{\nu}$. Then note that if $m=\sum_{\alpha} a_{\alpha} \bar{\partial}^{\alpha}$ then $m=Q \cdot 1$, where $Q=\sum_{\alpha} a_{\alpha} \partial^{\alpha}$. Thus $\xi=r Q \cdot(1 \otimes 1)$. Therefore we have an obvious surjective map $\varphi: \operatorname{gr} D \rightarrow \operatorname{gr}_{\Omega} N$ which takes 1 to $\overline{1 \otimes 1}$. Thus $\Omega$ is a good filtration on $N$.

Let $\xi=[m \otimes r] \in \Omega_{v} / \Omega_{v-1}$. For $i \leq g$, we have $X_{i} m \in \mathcal{F}_{\nu-1}$. Thus $X_{i}(m \otimes r)=$ $m \otimes X_{i} r=X_{i} m \otimes r \in \Omega_{v-1}$. Therefore $X_{i} \xi=0$ for all $i \leq g$. Also notice that for $i>g$, we have $\zeta_{i} \xi=\left[m \otimes \partial_{i}(r)\right]$. But $m \otimes \partial_{i} r \in \Omega_{v}$. As degree $\zeta_{i}=1$, we get that $\zeta_{i} \xi=0$. Thus $\varphi$ factors to a surjective map $\bar{\varphi}: \mathcal{O} /\left(X_{1}, \ldots, X_{g}\right)\left[\zeta_{1}, \ldots, \zeta_{g}\right] \rightarrow \operatorname{gr}_{\Omega} N$.

As $\operatorname{gr}_{\Omega} N$ has dimension $n$, it follows that $\bar{\varphi}$ is in fact an isomorphism. Thus $\operatorname{gr}_{\Omega} N \cong \mathcal{O} /\left(X_{1}, \ldots, X_{g}\right)\left[\zeta_{1}, \ldots, \zeta_{g}\right]$ and clearly it has multiplicity one.

We need the following result in the proof of our main result.

Corollary 7.8. Let $\mathcal{O}=K\left[\left[X_{1}, \ldots, X_{n}\right]\right]$ and let $P=\left(X_{1}, \ldots, X_{g}\right)$, where $g<n$. Set $\mathfrak{m}=\left(X_{1}, \ldots, X_{n}\right)$. Let $J \subseteq P$. Let $\theta^{g}: H_{P}^{g}(\mathcal{O}) \rightarrow H_{J}^{g}(\mathcal{O})$ be the natural map. Then Ass image $\theta^{g}$ is either empty or equal to $\{P\}$. In particular $\mathfrak{m} \notin$ Ass image $\theta^{g}$.

Proof. We note that any injective $D$-module is also an injective $\mathcal{O}$-module. Thus we can take an injective resolution of $\mathcal{O}$ as a $D$-module and note that computing $\theta^{i}$ with this resolution, we get that $\theta^{i}: H_{P}^{i}(\mathcal{O}) \rightarrow H_{J}^{i}(\mathcal{O})$ is $D$-linear. By Lemma 7.7, we get that $N=H_{P}^{g}(\mathcal{O})$ is a simple $D$-module. Thus image $\theta^{g} \cong N$ or it is zero. The result follows.

\section{Small dimensions}

In this section we prove several elementary results regarding local cohomology modules of regular rings of dimension $\leq 4$. We note that the results for dimension $\leq 3$ are probably well known to the experts. However, as we are unable to find a reference, we give a proof for these cases too.

We first prove the following general result.

Proposition 8.1. Let $R$ be a regular ring of dimension $d$. Let $I$ be an ideal in $R$. Then

$$
\operatorname{Ass}_{R}\left(H_{I}^{d}(R)\right)=\{P \mid P \in \operatorname{Min} R / I \text { and height } P=d\} .
$$

Proof. We may assume $I$ is a radical ideal. Suppose $P \in \operatorname{Ass}_{R}\left(H_{I}^{d}(R)\right)$. Then as height $P \geq d=\operatorname{dim} R$, we get that $P$ is a maximal ideal of $R$. Note that $P R_{P} \in \operatorname{Ass}_{R_{P}}\left(H_{I R_{P}}^{d}\left(R_{P}\right)\right)$ and thus $P \widehat{R_{P}} \in \operatorname{Ass}_{\widehat{R_{P}}}\left(H_{\overparen{R_{P}}}^{d}\left(\widehat{R_{P}}\right)\right)$.

Notice that $\widehat{R_{P}}$ is a domain and so by the Hartshorne-Lichtenbaum theorem (see [Iyengar et al. 2007, Theorem 14.1]), we get that $I \widehat{R_{P}}$ is a zero-dimensional ideal in $\widehat{R_{P}}$. It follows that $I R_{P}$ is a zero -dimensional ideal in $R_{P}$. As $I R_{P}$ is a radical ideal, we get that $I R_{P}=P R_{P}$. The result follows. 
If $M$ is an $R$-module then set

$$
\operatorname{Ass}_{R}^{i}(M)=\{P \mid P \in \text { Ass } M \text { and height } P=i\} .
$$

As an easy corollary to Proposition 8.1, we get this:

Corollary 8.2. Let $R$ be a regular ring of dimension $d$. Let $I$ be an ideal in $R$. Then

$$
\bigcup_{i \geq 0} \operatorname{Ass}_{R}^{i}\left(H_{I}^{i}(R)\right)=\operatorname{Min} R / I .
$$

Proof. We may assume $I$ is a radical ideal. Let $I=P_{1} \cap \cdots \cap P_{r}$. Notice that $I R_{P_{i}}=P_{i} R_{P_{i}}$ for each $i$ and $R_{P_{i}}$ is a regular local ring of height $c_{i}=$ height $P_{i}$. Notice that $P_{i} \in \operatorname{Ass}_{R}^{c_{i}}\left(H_{I}^{c_{i}}(R)\right)$. Thus

$$
\operatorname{Min} R / I \subseteq \bigcup_{i \geq 0} \operatorname{Ass}_{R}^{i}\left(H_{I}^{i}(R)\right) .
$$

Conversely let $P \in \operatorname{Ass}_{R}^{i}\left(H_{I}^{i}(R)\right)$. We localize at $P$. Then $R_{P}$ is a regular local ring of dimension $i$. The result now follows from Proposition 8.1.

Remark 8.3. If $I$ is an ideal of height $g$ then it is well known that

$$
\text { Ass } H_{I}^{g}(R)=\{P \mid P \supseteq I \text {, height } P=g\} \text {. }
$$

We now prove this:

Lemma 8.4. Let $R$ be a regular ring and let $I$ be an ideal of height one. Then $\operatorname{Ass}_{R} H_{I}^{i}(R)$ is finite for $i=1,2$.

Proof. For $i=1$, the result follows from Remark 8.3.

For $i=2$, we first consider the case when $I$ has a primary decomposition $I=Q_{1} \cap \cdots \cap Q_{r}$, where height $\sqrt{Q_{i}}=1$ for all $i$. We claim that $H_{I}^{j}(R)=0$ for all $j \geq 2$. Suppose this is not true. Let $P \in \operatorname{Ass} H_{I}^{j}(R)$ for some $j \geq 2$. We localize at $P$. We now note that $I R_{P}$ is a principal ideal; see [Bruns and Herzog 1993, Exercise 2.2.28]. So $H_{I R_{P}}^{s}\left(R_{P}\right)=0$ for all $s \geq 2$, a contradiction. Therefore $H_{I}^{j}(R)=0$ for all $j \geq 2$. Thus our assertion holds in this special case.

Now let $I$ be a general ideal of height one. Then $I=J \cap K$, where $K$ has height $\geq 2$ and $J$ is an ideal of height one. Furthermore, $J$ is of the special kind discussed above. So $H_{J}^{j}(R)=0$ for $j \geq 2$. By the Mayer-Vietoris sequence (see [Iyengar et al. 2007, Theorem 15.1]) and noting that height $(J+K) \geq 3$, we have an exact sequence

$$
0 \rightarrow H_{K}^{2}(R) \rightarrow H_{I}^{2}(R) \rightarrow H_{J+K}^{3}(R) .
$$

As height $K \geq 2$ and height $(J+K) \geq 3$, the result follows from Remark 8.3.

An easy consequence of the above results is the following: 
Corollary 8.5. Let $R$ be a regular ring of dimension $d \leq 3$. Then for any ideal $I$, we have Ass $H_{I}^{i}(R)$ is a finite set for all $i \geq 0$.

Proof. By Remark 2.16, we may assume that $R$ is a domain. We have nothing to show for $d=0$. The assertion for $d=1$ follows from Proposition 8.1. For $d=2$, the result follows from Lemma 8.4 and Remark 8.3.

Now consider the case when $d=3$. If height $I=1$ then the result follows from Lemma 8.4 and Proposition 8.1. If height $I=2$, the result follows from Remark 8.3 and Proposition 8.1. If height $I=3$, the result follows from Proposition 8.1.

We now give a proof of Corollary 1.6.

Proof. By Corollary 8.5, we may assume $\operatorname{dim} R=4$. By the results of [Huneke and Sharp 1993], the result holds when char $K=p>0$. Thus we may assume that char $K=0$. By Remark 2.16, we may assume that $R$ is a domain.

If height $I=1$, then the result follows from Lemma 8.4, Theorem 1.3 and Proposition 8.1. If height $I=2$, the result follows from Remark 8.3, Theorem 1.3 and Proposition 8.1. If height $I=3$, the result follows from Remark 8.3 and Proposition 8.1. If height $I=4$, the result follows from Proposition 8.1.

\section{Proofs of Corollary 1.7 and Proposition 1.10}

In this final section we give an application of Theorem 1.3. Throughout $R$ will denote a regular ring of dimension $d$ containing a field of characteristic zero.

Proof of Corollary 1.7. Suppose, if possible, that

$$
\bigcup_{i \geq 0} \operatorname{Ass}_{R}^{i+1}\left(H_{I}^{i}(R)\right) \quad \text { is an infinite set. }
$$

As $H_{I}^{i}(R)=0$ for $i>d$, we get that $\operatorname{Ass}_{R}^{i+1}\left(H_{I}^{i}(R)\right)$ is infinite for some $i \leq d$. By Corollary 3.7, we may assume that $R$ contains an uncountable field. Suppose $\operatorname{Ass}_{R}^{i+1}\left(H_{I}^{i}(R)\right)=\left\{\mathfrak{p}_{n}\right\}_{n \geq 1}$. Consider the ring

$$
A=S^{-1} R, \quad \text { where } S=R \backslash \bigcup_{n \geq 1} \mathfrak{p}_{n} .
$$

Then $A$ is a regular ring of dimension $i+1$. Furthermore, $\operatorname{Ass}_{A} H_{I A}^{i}(A)$ is an infinite set. This contradicts Theorem 1.3.

Recall that if $(A, \mathfrak{m})$ is a local ring then $\operatorname{Spec}^{\circ}(A)=\operatorname{Spec}(A) \backslash\{\mathfrak{m}\}$ considered as a subspace of $\operatorname{Spec}(A)$.

Definition 9.1. Let $(A, \mathfrak{m})$ be a local ring and let $I$ be an ideal in $A$. We say $\operatorname{Spec}^{\circ}(A / I)$ is absolutely connected if for every flat local map $(A, \mathfrak{m}) \rightarrow(B, \mathfrak{n})$ with $\mathfrak{m} B=\mathfrak{n}$ and $B / \mathfrak{n}$ algebraically closed, $\operatorname{Spec}^{\circ}(B / I B)$ is connected. 
Remark 9.2. It is easy to see that if $\operatorname{Spec}^{\circ}(A / I)$ is absolutely connected then it is connected.

Proof of Proposition 1.10. First assume that $P \supseteq I$, height $P=c \geq g+2$ and $\operatorname{Spec}^{\circ}\left(R_{P} / I_{P}\right)$ is not absolutely connected. So there is a flat extension $(B, \mathfrak{n})$ of $R_{P}$ such that $B$ is complete, $P B=\mathfrak{n}, B / \mathfrak{n}$ algebraically closed and $\operatorname{Spec}^{\circ}(B / I B)$ is disconnected. We note that $B$ is a complete regular ring of dimension $c$. Also note that as $\operatorname{dim} R_{P} / I_{P} \geq 2$, we have that $\operatorname{dim} B / I B \geq 2$. By the result of Ogus [1973, Corollary 2.11], we have that $H_{I B}^{c-1}(B) \neq 0$. So $H_{I R_{P}}^{c-1}\left(R_{P}\right) \neq 0$. Therefore $P \in \operatorname{Supp} H_{I}^{c-1}(R)$. We claim that $P$ is a minimal prime of $H_{I}^{c-1}(R)$. Suppose there exists $Q \in \operatorname{Supp} H_{I}^{c-1}(R)$ and $Q \subsetneq P$. Then height $Q \leq c-1$. By Grothendieck's vanishing theorem, we have height $Q \geq c-1$. So height $Q=c-1$. By our assumption, we have that $\operatorname{dim} R_{Q} / I R_{Q} \geq 1$. So $\operatorname{dim} \widehat{R_{Q}} / I \widehat{R_{Q}} \geq 1$. By the Hartshorne-Lichtenbaum theorem, we have $H_{I \widehat{R_{Q}}}^{c-1}\left(\widehat{R_{Q}}\right)=0$. Thus

$$
\left(H_{I}^{c-1}(R)\right)_{Q}=H_{I R_{Q}}^{c-1}\left(R_{Q}\right)=0,
$$

a contradiction. Therefore $P$ is a minimal prime of $H_{I}^{c-1}(R)$ and so belongs to $\operatorname{Ass}^{c}\left(H_{I}^{c-1}(R)\right)$.

Conversely assume $P \in \operatorname{Ass}^{c}\left(H_{I}^{c-1}(R)\right)$. So $H_{I R_{P}}^{c-1}\left(R_{P}\right) \neq 0$. By Remark 8.3, we get that $c-1 \geq g+1$. So $c \geq g+2$. Let $R_{P} \rightarrow B$ be any local flat extension with $(B, \mathfrak{n})$ complete, $P B=\mathfrak{n}$ and $B / \mathfrak{n}$ algebraically closed. We note that by our assumptions, $\operatorname{dim} R_{P} / I R_{P} \geq 2$. As $R_{P} / I R_{P} \rightarrow B / I B$ is flat local map with fiber a field, we have that $\operatorname{dim} B / I B=\operatorname{dim} R_{P} / I R_{P} \geq 2$. Also by faithful flatness, $H_{I B}^{c-1}(B) \neq 0$. Thus again by the same result of $\operatorname{Ogus,} \operatorname{Spec}^{\circ}(B / I B)$ is disconnected. Therefore $\operatorname{Spcc}^{\circ}\left(R_{P} / I_{P}\right)$ is not absolutely connected.

Remark 9.3. The above result is also true in characteristic $p$ with the same proof. The reason is that Ogus' result is true in characteristic $p$; see [Peskine and Szpiro 1973, Theorem III.5.5].

\section{References}

[Bhatt et al. 2014] B. Bhatt, M. Blickle, G. Lyubeznik, A. K. Singh, and W. Zhang, "Local cohomology modules of a smooth $\mathbb{Z}$-algebra have finitely many associated primes", Invent. Math. 197:3 (2014), 509-519. MR 3251828 Zbl 1318.13025

[Björk 1979] J.-E. Björk, Rings of differential operators, North-Holland Mathematical Library 21 , North-Holland, Amsterdam, 1979. MR 82g:32013 Zbl 0499.13009

[Brodmann and Sharp 1998] M. P. Brodmann and R. Y. Sharp, Local cohomology: an algebraic introduction with geometric applications, Cambridge Studies in Advanced Mathematics 60, Cambridge University Press, 1998. MR 99h:13020 Zbl 0903.13006

[Brodmann et al. 2000] M. P. Brodmann, C. Rotthaus, and R. Y. Sharp, "On annihilators and associated primes of local cohomology modules", J. Pure Appl. Algebra 153:3 (2000), 197-227. MR 2002b:13027 Zbl 0968.13010 
[Bruns and Herzog 1993] W. Bruns and J. Herzog, Cohen-Macaulay rings, Cambridge Studies in Advanced Mathematics 39, Cambridge University Press, 1993. 2nd edition published in 1998. MR 95h:13020 Zbl 0788.13005

[Huneke and Lyubeznik 1990] C. L. Huneke and G. Lyubeznik, "On the vanishing of local cohomology modules”, Invent. Math. 102:1 (1990), 73-93. MR 91i:13020 Zbl 0717.13011

[Huneke and Sharp 1993] C. L. Huneke and R. Y. Sharp, "Bass numbers of local cohomology modules", Trans. Amer. Math. Soc. 339:2 (1993), 765-779. MR 93m:13008 Zbl 0785.13005

[Iyengar et al. 2007] S. B. Iyengar, G. J. Leuschke, A. Leykin, C. Miller, E. Miller, A. K. Singh, and U. Walther, Twenty-four hours of local cohomology, Graduate Studies in Mathematics 87, American Mathematical Society, Providence, RI, 2007. MR 2009a:13025 Zbl 1129.13001

[Lyubeznik 1993] G. Lyubeznik, "Finiteness properties of local cohomology modules (an application of D-modules to commutative algebra)", Invent. Math. 113:1 (1993), 41-55. MR 94e:13032 Zbl 0795.13004

[Lyubeznik 1997] G. Lyubeznik, " $F$-modules: applications to local cohomology and $D$-modules in characteristic $p>0$ ", J. Reine Angew. Math. 491 (1997), 65-130. MR 99c:13005 Zbl 0904.13003

[Lyubeznik 2000] G. Lyubeznik, "Finiteness properties of local cohomology modules for regular local rings of mixed characteristic: the unramified case", Comm. Algebra 28:12 (2000), 5867-5882. MR 2002b:13028 Zbl 0981.13008

[Lyubeznik 2002] G. Lyubeznik, "A partial survey of local cohomology”, pp. 121-154 in Local cohomology and its applications (Guanajuato, 1999), edited by G. Lyubeznik, Lecture Notes in Pure and Applied Mathematics 226, Dekker, New York, 2002. MR 2003b:14006 Zbl 1061.14005

[Marley 2001] T. Marley, "The associated primes of local cohomology modules over rings of small dimension”, Manuscripta Math. 104:4 (2001), 519-525. MR 2002h:13027 Zbl 0987.13009

[Matsumura 1989] H. Matsumura, Commutative ring theory, 2nd ed., Cambridge Studies in Advanced Mathematics 8, Cambridge University Press, 1989. MR 90i:13001 Zbl 0666.13002

[Ogus 1973] A. Ogus, "Local cohomological dimension of algebraic varieties", Ann. of Math. (2) 98 (1973), 327-365. MR 58 \#22059 Zbl 0308.14003

[Peskine and Szpiro 1973] C. Peskine and L. Szpiro, "Dimension projective finie et cohomologie locale: applications à la démonstration de conjectures de M. Auslander, H. Bass et A. Grothendieck", Inst. Hautes Études Sci. Publ. Math. 42 (1973), 47-119. MR 51 \#10330 Zbl 0268.13008

[Rotthaus 1980] C. Rotthaus, "Zur Komplettierung ausgezeichneter Ringe”, Math. Ann. 253:3 (1980), 213-226. MR 82c:13023 Zbl 0444.13007

Received June 10, 2015. Revised August 20, 2015.

TONY J. PuthenPuRAKal

DEPARTMENT OF MATHEMATICS

INDIAN INSTITUTE OF TECHNOLOGY BOMBAY

POWAI

MUMBAI 400076

INDIA

tputhen@gmail.com 


\title{
PACIFIC JOURNAL OF MATHEMATICS
}

\author{
msp.org/pjm
}

Founded in 1951 by E. F. Beckenbach (1906-1982) and F. Wolf (1904-1989)

\section{EDITORS}

Don Blasius (Managing Editor)

Department of Mathematics

University of California

Los Angeles, CA 90095-1555

blasius@math.ucla.edu

\author{
Paul Balmer \\ Department of Mathematics \\ University of California \\ Los Angeles, CA 90095-1555 \\ balmer@math.ucla.edu \\ Robert Finn \\ Department of Mathematics \\ Stanford University \\ Stanford, CA 94305-2125 \\ finn@math.stanford.edu \\ Sorin Popa \\ Department of Mathematics \\ University of California \\ Los Angeles, CA 90095-1555 \\ popa@math.ucla.edu
}

\author{
Vyjayanthi Chari \\ Department of Mathematics \\ University of California \\ Riverside, CA 92521-0135 \\ chari@math.ucr.edu \\ Kefeng Liu \\ Department of Mathematics \\ University of California \\ Los Angeles, CA 90095-1555 \\ liu@math.ucla.edu \\ Jie Qing \\ Department of Mathematics \\ University of California \\ Santa Cruz, CA 95064 \\ qing@ cats.ucsc.edu
}

\section{PRODUCTION}

Silvio Levy, Scientific Editor, production@msp.org

\section{SUPPORTING INSTITUTIONS}

ACADEMIA SINICA, TAIPEI

CALIFORNIA INST. OF TECHNOLOGY

INST. DE MATEMÁTICA PURA E APLICADA

KEIO UNIVERSITY

MATH. SCIENCES RESEARCH INSTITUTE

NEW MEXICO STATE UNIV.

OREGON STATE UNIV.

\author{
STANFORD UNIVERSITY \\ UNIV. OF BRITISH COLUMBIA \\ UNIV. OF CALIFORNIA, BERKELEY \\ UNIV. OF CALIFORNIA, DAVIS \\ UNIV. OF CALIFORNIA, LOS ANGELES \\ UNIV. OF CALIFORNIA, RIVERSIDE \\ UNIV. OF CALIFORNIA, SAN DIEGO \\ UNIV. OF CALIF., SANTA BARBARA
}

\author{
Daryl Cooper \\ Department of Mathematics \\ University of California \\ Santa Barbara, CA 93106-3080 \\ cooper@math.ucsb.edu \\ Jiang-Hua Lu \\ Department of Mathematics \\ The University of Hong Kong \\ Pokfulam Rd., Hong Kong \\ jhlu@maths.hku.hk \\ Paul Yang \\ Department of Mathematics \\ Princeton University \\ Princeton NJ 08544-1000 \\ yang@math.princeton.edu
}

These supporting institutions contribute to the cost of publication of this Journal, but they are not owners or publishers and have no responsibility for its contents or policies.

See inside back cover or msp.org/pjm for submission instructions.

The subscription price for 2016 is US $\$ 440 /$ year for the electronic version, and $\$ 600 /$ year for print and electronic.

Subscriptions, requests for back issues and changes of subscribers address should be sent to Pacific Journal of Mathematics, P.O. Box 4163, Berkeley, CA 94704-0163, U.S.A. The Pacific Journal of Mathematics is indexed by Mathematical Reviews, Zentralblatt MATH, PASCAL CNRS Index, Referativnyi Zhurnal, Current Mathematical Publications and Web of Knowledge (Science Citation Index).

The Pacific Journal of Mathematics (ISSN 0030-8730) at the University of California, c/o Department of Mathematics, 798 Evans Hall \#3840, Berkeley, CA 94720-3840, is published twelve times a year. Periodical rate postage paid at Berkeley, CA 94704, and additional mailing offices. POSTMASTER: send address changes to Pacific Journal of Mathematics, P.O. Box 4163, Berkeley, CA 94704-0163.

PJM peer review and production are managed by EditFLOW ${ }^{\circledR}$ from Mathematical Sciences Publishers.

\section{PUBLISHED BY}

\section{mathematical sciences publishers \\ nonprofit scientific publishing}

http://msp.org/

(C) 2016 Mathematical Sciences Publishers 


\section{PACIFIC JOURNAL OF MATHEMATICS}

Volume 282 No. $1 \quad$ May 2016

On the half-space theorem for minimal surfaces in Heisenberg space

Tristan Alex

Extending smooth cyclic group actions on the Poincaré homology sphere 9

NIMA ANVARI

A short proof of the existence of supercuspidal representations for all reductive $p$-adic groups

RAPHAËL BEUZART-PLESSIS

Quantum groups and generalized circular elements

MICHAEL BRANNAN and KAY KIRKPATRICK

Volumes of Montesinos links

KathleEn Finlinson and Jessica S. PurCell

Minimal surfaces with two ends which have the least total absolute curvature

SHOICHI FUJIMORI and TOSHIHIRO SHODA

Multiplicité du spectre de Steklov sur les surfaces et nombre chromatique

\section{PIERRE JAMMES}

$E$-polynomial of the $\mathrm{SL}(3, \mathbb{C})$-character variety of free groups

SEAN LAWTON and VicEnTE MuÑOZ

The Blum-Hanson property for $\mathscr{C}(K)$ spaces

PASCAL LEFÈVRE and ÉTIENNE MATHERON

Crossed product algebras and direct integral decomposition for Lie supergroups

KARL-HERMANN NEEB and HADI SALMASIAN Associated primes of local cohomology modules over regular rings

TONY J. PUTHENPURAKAL 